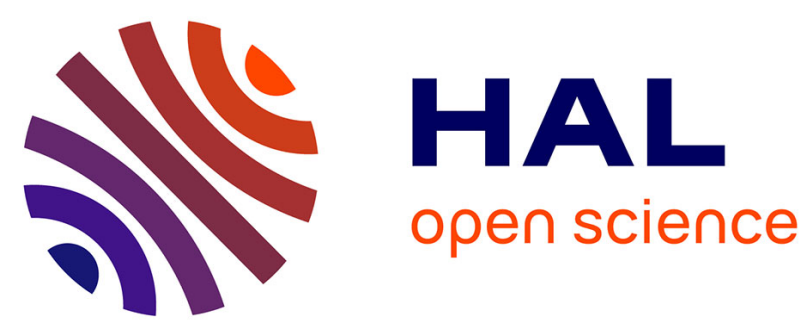

\title{
SAMOS for Spatial Architecture based on Multi- physics and Organisation of Systems in conceptual design
}

\author{
Romain Barbedienne, Yethreb Ben Messaoud, Jean-Yves Choley, Olivia \\ Penas, Achour Ouslimani, Alain Riviere
}

\section{- To cite this version:}

Romain Barbedienne, Yethreb Ben Messaoud, Jean-Yves Choley, Olivia Penas, Achour Ouslimani, et al.. SAMOS for Spatial Architecture based on Multi- physics and Organisation of Systems in conceptual design. Systems Engineering (ISSE), 2015 IEEE International Symposium on, Sep 2015, Rome, Italy. pp.135-141, 10.1109/SysEng.2015.7302746 . hal-01591527

\section{HAL Id: hal-01591527 \\ https://hal.science/hal-01591527}

Submitted on 21 Sep 2017

HAL is a multi-disciplinary open access archive for the deposit and dissemination of scientific research documents, whether they are published or not. The documents may come from teaching and research institutions in France or abroad, or from public or private research centers.
L'archive ouverte pluridisciplinaire HAL, est destinée au dépôt et à la diffusion de documents scientifiques de niveau recherche, publiés ou non, émanant des établissements d'enseignement et de recherche français ou étrangers, des laboratoires publics ou privés. 


\title{
SAMOS for Spatial Architecture based on Multi- physics and Organisation of Systems in conceptual design
}

\author{
Conference Paper · September 2015
}

DOI: 10.1109/SysEng.2015.7302746

\section{CITATION}

1

6 authors, including:

\section{READS}

120

\section{Olivia Penas}

Supméca - Institut supérieur de mécanique d... 33 PUBLICATIONS 173 CITATIONS

SEE PROFILE

\section{Achour Ouslimani}

École Nationale Supérieure de l'Electronique.. 80 PUBLICATIONS 219 CITATIONS SEE PROFILE

\section{Jean-Yves Choley}

Supméca - Institut supérieur de mécanique d... 84 PUBLICATIONS 216 CITATIONS

SEE PROFILE

\section{Alain Riviere}

Centre François Baclesse

92 PUBLICATIONS 2,076 CITATIONS

SEE PROFILE

Some of the authors of this publication are also working on these related projects: 


\title{
SAMOS for Spatial Architecture based on Multi- physics and Organisation of Systems in conceptual design
}

\author{
Romain Barbedienne ${ }^{1,2}$, Yethreb Ben Messaoud ${ }^{1}$, Jean-Yves Choley ${ }^{1,2}$, Olivia Penas ${ }^{1,2}$, Achour Ouslimani $^{1}$ and Alain \\ Rivière $^{1}$ \\ ${ }^{1}$ Laboratoire QUARTZ \\ Saint Ouen, France, \\ firstname.name@supmeca.fr \\ achour.ouslimani@ensea.fr \\ ${ }^{2}$ SIM Project \\ IRT SystemX \\ Palaiseau, France \\ firstname.name@irt-systemx.fr
}

\begin{abstract}
The conceptual design is a decisive phase where the simulation teams would like to quickly pre-validate spatial architectures from the physical architecture proposed by the system architects. In order to support them to efficiently achieve this task while meeting various industrial requirements, three approaches were proposed and compared, and finally the last and innovative approach called SAMOS (Spatial Architecture based on Multi-physics and Organization of Systems) is presented and described. The corresponding platform will allow to validate the spatial allocation of components under geometrical and multi-physical constraints, while facilitating the collaboration between the different design actors during the conceptual design.
\end{abstract}

Keywords-MBSE, Conceptual Design, System Architects, Geometers, architecture assesment, Multiphysical modeling, spatial allocation.

\section{INTRODUCTION}

\section{A. Industrial context and challenges}

The design cycle is usually divided into 4 main phases: the clarification of the task, the conceptual design, the embodiment design and the detailed design according to Guideline VDI 2221 [1]. Our research work focuses on the second phase which is the conceptual design.

In the conceptual design, the first step consists in analyzing users' needs and generating the requirements specification. According to these derived requirements, System Architects (SAs) provide a functional and a logical architecture. Then they propose different physical architecture alternatives of concepts to be assessed. Once one concept has been selected, designers perform preliminary and detailed designs. Finally, verification of derived requirements is achieved by SAs through the analysis of the geometrical and multi-physical detailed simulations.

Concerning the industrial practices regarding the assessment of concepts, designers usually start from a physical architecture provided by the SAs at the end of the logical design. Then, the Geometers (Gs), allocate a volume and an initial position of each component within the whole system relying on their expertise and field of knowledge without taking into account the opinions of other discipline-teams. Then, performances and spatial requirements are provided to the Simulation Teams (STs), composed of different discipline (electronics, mechanics, control, hardware/software ...) experts in charge of verifying that this physical architecture behavior meets them through general analytical simulations.

If some of these requirements are not satisfied, a long iterative costly process is performed until convergence to a suitable satisfactory architecture is fulfilled.

Due to high competition between companies, it is important to decrease design time in order to minimize the cost of product design. Moreover, the increasing complexity of systems implies high interactions between different disciplines that have to be taken into account. Thus, the introduction of a MBSE (Model-Based Systems Engineering) approach in the design life cycle of companies should meet these objectives.

\section{B. A MBSE design problematic}

The dramatic increase of design costs and time usually takes place during the embodiment or detailed design phases. This issue is, as a rule, related to the numerous and long iterations between different discipline technical services. Indeed, their respective physical behavior simulations are not always consistent, due to the lack of data uniformity between STs and the difficulty for them to have design collaborations using different non-interoperable discipline tools.

Therefore, we propose to study what would be the best MBSE approach that will not only ensure the consistency and traceability of data and models, but will also take into account geometrical and multi-physical constraints at the conceptual design phase. This would then enable SAs to more efficiently select concept architecture. The preliminary assessment of architectures under geometrical and physical constraints will thus reduce the risk of late changes during further design phases, and then the design time.

To address this objective, the proposed approaches have to meet the following requirements related to the conceptual phase: simulations have to be faster, based on simplified 
geometry of components (not yet well defined), in order to give orders of magnitude (approximated results) allowing to compare different potential architectures of concept. Moreover, instead of making only performance simulations, that slightly take into account geometrical considerations, adding simulations of multi-physical behaviors would allow to consider multi-physics for architecture assessment, and then reduce the design risk of integrated multi-domain systems, such as mechatronic ones, Cyber-Physical Systems, etc.

Moreover, MBSE approaches allow not only to increase the collaboration and consistency successively between teams and models, but also to improve the traceability with requirements and to promote the reuse of the models.

Finally, to meet all these objectives, this paper deals with some alternative MBSE approaches, based on model transformations between system models, physical behavior simulation models and 3D models. These approaches aim at evaluating physical architectures during the conceptual design phase, relying on preliminary analytical multi-physical simulations under geometrical constraints.

\section{RELATED WORKS}

Currently, model transformation is a very popular approach to manage interoperability between different actors of the design cycle [2] [3]. The emergence of many recent languages specialized in model transformation, like ATL [4], M2M [3], MOF [2], QVT [2], points out the interest of such an approach, notably for research studies.

There are two kinds of transformation: from a model to a model (M2M), and from a model to a text (M2T). Throughout the following paper, M2M will then mean "model to model" transformation. While M2M transformation is rather a means to ensure modeling semantic interoperability, M2T transformation is rather used to automatically generate text documents from models to improve collaborations through a MBSE (and not document-based) process. M2T transformations then enable all design process actors to easily understand the content of a model without necessarily knowing the modeling language, even if this kind of transformations slows down the design process, since later new models based on generated documents have still to be developed.

A common approach to transform System models, implemented in SysML (System Modeling Language) or UML (Unified Modeling Language), into another language or a tool, is to enrich UML and SysML by using UML profiles or SysML extensions, which add the STs required modeling elements to the System model. In fact, UML profiles allow to customize the UML meta-model for particular domains. An extension consists in refining standard semantics of a UML profile in a strictly additive manner. Profiles and extensions define then stereotypes, tag definitions and constraints which are applied to specific model elements (meta-class) such as classes, attributes, operations and activities.

In order to operate the corresponding model transformation between SysML/UML, simulation and 3D models, many profiles have already been developed in order to support it. The profiles that address the link between SysML/UML and simulation models are often developed by transforming the semantics of a simulation tool into the SysML or UML semantics. Some examples of model transformations starting from SysML are cited below. A first SysML extension, based on standards, supports bond graphs analysis [5], and enables the transformation of system models into Modelica, Simulink, or other 0D solvers. A second profile proposed by Cao et al. addresses the model transformation from a System model in extended SysML into Simulink [6], but requires to preliminary modify the whole SysML model. Another model transformation between extended SysML model and the TRNSYS commercial software enables the performance evaluation of thermal and electrical energy systems [7]. The model transformation between SysML and DEVS (Discrete Event System Specification) formalism [8] describes state transitions and differential equations of the system, but does not permit to simulate. Finally, the two most well-known profiles SysML4Modelica [9] and ModelicaML [10] link SysML to Modelica, using their semantic similarity [9] and their common acausale modeling. ModelicaML, the first UML profile linking UML with Modelica, includes Modelica elements in UML but the whole Modelica program has to be implemented in UML. Thus, STs need to use UML before transferring the model into Modelica, even if UML is not convenient for programming whereas SAs, who is usually familiar with UML is not specifically able to model multiphysics. For this reason, Schamai et al. propose another model transformation based on a SysML extension named SysML4Modelica [9], that integrates Modelica modeling in SysML, and Modelica code is automatically generated from a diagram in SysML and does not require to be directly written in SysML. Nevertheless, SysML4Modelica does not take into account the definition of components geometry needed by STs, to generate a 3D architecture of components with multiphysical requirements

The inconvenient of such approaches, is that the developed SysML extensions for simulation modeling have to be used by STs that are not comfortable with this language. Moreover, the geometry modeling which is an important part of the conceptual design is also often forgotten in all previous ones.

In parallel, some authors have already worked with a MBSE approach to introduce the geometrical point of view in the System model. Baysal et al. proposed a method for geometrical modeling and positioning, related to the tolerance analysis in UML [11]. Nonetheless, the considered positioning was not relative and did not directly integrate any constraints, making it really difficult for designers to calculate general positioning of each part. Besides, Albers et al. propose the Contact \& Channel-Approach [12] to build Contact \& Channel-Models [13] through a SysML extension [14]. This modeling defines Working Surface Pairs as interface surfaces that are connected by physical components or volumes of liquids, gases or spaces, named Channel and Support Structures [13]. As their objective is to represent engineering artefacts when taking into account physical flows between components, their geometry modeling is only based on the working/ interacting surfaces, and does not allow to generate either the whole volume of components or relative positioning constraints. Finally, Böhnke et al. [15] propose a UML profile 
that defines the 3D geometry of components, but without managing assembly constraints of components: components are created by linking different sections represented by points. This method is in fact more adapted to complex detailed geometries and then not useful for conceptual design.

Finally, none of these studies allows to integrate the whole model transformation process from System, geometry and simulation models. Consequently, in order to tackle this issue, we propose three different approaches, that respect the actors' competencies and know-how by providing a collaborative framework relying on a MBSE approach to support architecture assessment.

\section{DIFFERENT APPROACHES FOR CONCEPTUAL ASSESSMENT WITH GEOMETRICAL AND PHYSICAL CONSTRAINTS}

Since the traditional methods of system architecture assessment during the conceptual design phase don't satisfy the industrial MBSE expectations, we propose three new approaches which will be successively described in this section. These approaches aim at ensuring the suitability of the exchanged models during the different conceptual design stages through some successive model transformations respectively between the System Architects' (SAs), the Geometers' (Gs) and the Simulation Teams' (STs) models. Indeed, in order to make the architecture assessment more efficient and reduce the late iterative process, multi-physical simulations, usually taken during the embodiment design phase, are then put forward in the conceptual design phase. In this way, it is important to note that the components geometry will be simplified and the simulation will be analytical, in order to meet the conceptual design needs previously described.

\section{A. First approach for design interactions management in a new architecture platform}

The first suggested approach illustrated in Fig. 1 presents a modification of the common industrial practices, presented in the section I.A, for the conceptual design. This approach proposes to provide a "Multi-physical architecture platform" aiming at facilitating the design interactions management between the different actors (SAs, Gs and STs).

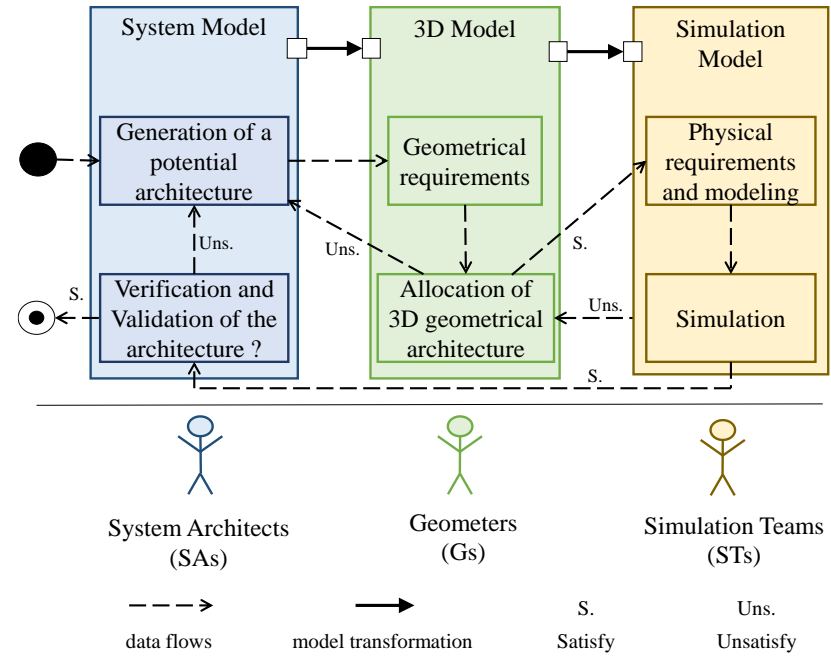

Fig. 1 Principles of the first multi-physical architecture platform
The whole process is described below. Firstly, the multiphysical architecture platform will allow for SAs to provide Gs the physical architecture, to be assessed, that will be automatically transformed from a System model into a 3D model, in order Gs specifies the allocation of components volume. This is ensured by a System-3D model transformation, which will generate, for each component, an empty 3D modeling element. In the new generated 3D model, Gs will first add geometrical requirements, and then assign the geometry (form and volume) and an initial position to each component of the whole system. Secondly, the platform will provide the model transformation from the predefined 3D model (completed by the Gs) into a simulation model. This M2M transformation takes into account the architecture components with their corresponding geometrical data. Then STs add the physical requirements before performing analytical simulations in order to verify whether this 3D designed architecture meets these requirements and the spatial allocation resulting from the Gs. Usually, at the beginning of this process, the first 3D architecture proposed by the Gs does not meet all the physical performance requirements provided by the SAs, thus STs request Gs to make some modifications on their spatial allocation or to give some degree of freedom on components geometry or relative position constraints. Consequently, the iterations can remain numerous until a convenient architecture is found, and for each iteration STs have to realize a new physical modeling of the last proposed 3D spatial architecture. If the problem persists, where STs do not succeed in meeting physical performances with any proposed spatial allocation from Gs, the potential physical architecture is invalidated by the SAs who have to propose another physical architecture. After that, the iterative previous steps restart and the different actors are confronted with the same costly and long process.

Concerning the advantages of this first approach, it ensures a slight improvement of design interactions between the different actors, by automatically transferring, by successive model transformations, the specifications necessary for the assessment process, instead of oral or documents exchanges. The multi-physical platform gives design actors an interactive support by gathering all design information and facilitating the automatic data exchange of the different models in real time during the architecture assessment process. However, even if the number of iterations is approximately as high as the common approach, which can prove very costly for companies and generate some time losses, this first approach can improve the consistency of design data through model transformation processes between System model (in SysML), geometry model (3D model) and simulation model. Moreover, the traceability, in the system model, of geometrical and physical data resulting from the assessment process cannot be ensured, because System model usually does not contain the corresponding semantics.

Therefore, it is now essential to make a quicker architecture assessment, which would be more efficient and would then feature numerous cost and time benefits, while providing modeling data traceability.

\section{B. Second Approach: geometrical and multi-physical enrichments for semantic interoperability}


The second suggested approach, illustrated in Fig. 2, proposes to enrich System model with geometrical and physical semantics, in order to add the traceability process required by MBSE through bidirectional model transformations.

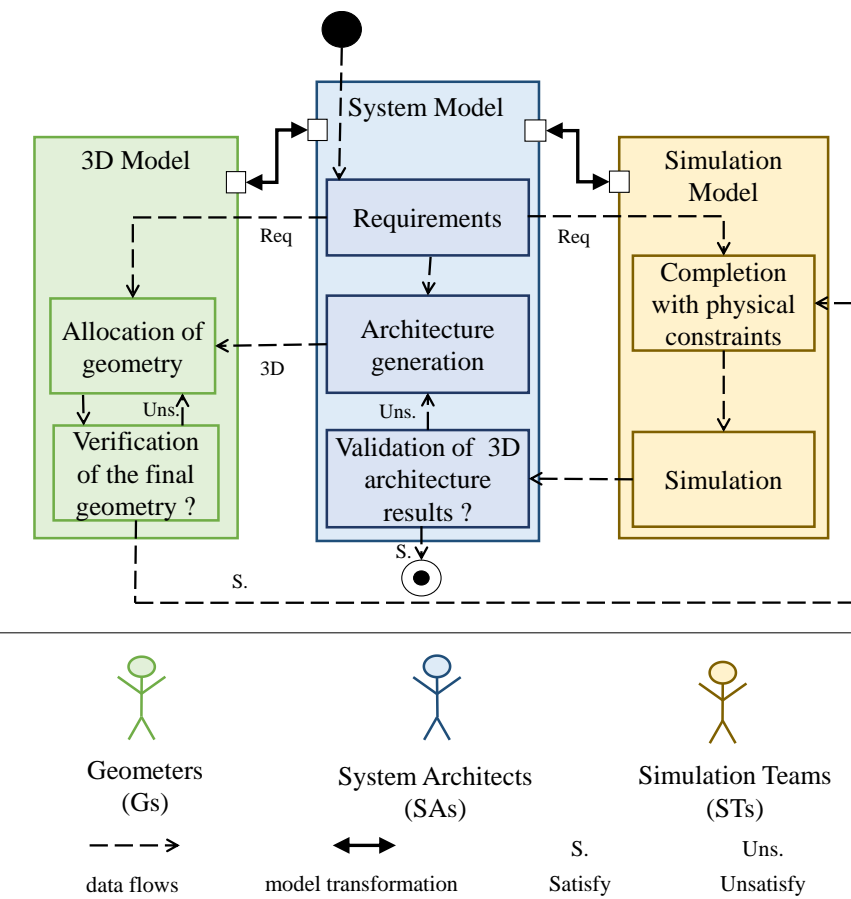

Fig. 2 Principles of the second multi-physical architecture platform

The process of this approach is slightly different from the first one, since the SAs can generate architectures with geometrical and multi-physical requirements, thanks to the System models semantics enrichments. Then a model transformation takes place and provides, for each architecture component, the corresponding 3D modeling element, integrating geometrical requirements (form and volume for example) previously specified by the SAs in the System model. In accordance with other geometrical requirements (positioning constraints for example), Gs complete the remaining components volume allocation and position, in their 3D environment tool. Once this 3D complete architecture has been done, the reverse model transformation from the 3D model into the System model allows to enrich the System model with geometrical information from this 3D architecture. This geometry-enriched physical architecture and physical requirements are then provided to STs through another model transformation in order to be assessed by some quick analytical physical simulations. Depending on these simulation results, if they fulfill SAs' requirements, this physically-enriched 3D architecture is traced back to the System model by a third model transformation to be validated by SAs. On the contrary, if the simulation results do not satisfy the initial SAs' requirements, they are still traced back to the System model for capitalization, and SAs have to decide if they prefer to ask Gs for another 3D alternative architecture or to propose another physical architecture, before restarting the assessment process. After a number of iterations, the process converges to an enriched system architecture satisfying the different geometrical and physical requirements.

The first advantage of this approach is to reduce the number of design iterations. Both Gs and STs can indeed themselves verify if the enriched 3D proposed architecture meets respectively the geometrical and physical requirements that were initially given to them by SAs, and this before tracing back the results of their work in the System model for SAs validation. Then, the semantic geometrical and physical enrichments of the System model allow to trace and capitalize the geometrical and physical information of the assessed 3D architecture in the System model, so that SAs have all the necessary information in a single model to validate or not the proposed 3D architecture. Finally, bidirectional model transformations prove to be quick processes to ensure the consistency and the traceability of models specifications and results. All these advantages contribute to reducing design time and costs for the concept assessment process.

However, this approach is not efficient enough, since for each architecture modification, the proposed process requires SAs' intervention. Indeed, as this platform provides an access to the whole design information only through the System model, it does not allow Gs and STs to interact to quickly find a compromise that fulfills the SAs' requirements.

\section{Third approach: SAMOS}

To tackle this problem, a third approach presented in Fig. 3, proposes to integrate Gs' and STs' tasks within a single 3D multi-physical modeler environment.

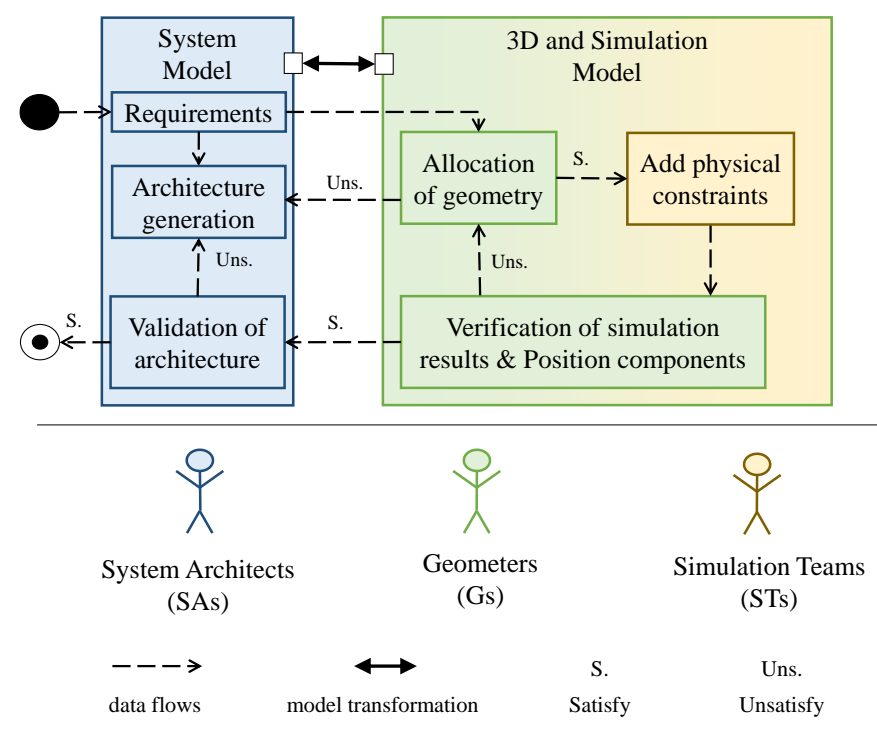

Fig. 3 Principles of the third multi-physical architecture platform (SAMOS)

This process begins with the generation by the SAs of the physical architecture and the geometrical and physical requirements that are automatically transferred into a $3 \mathrm{D}$ and simulation model, through the model transformation process. Then, Gs achieve the initial components volume allocation that meets the previous SAs' geometrical requirements in the $3 \mathrm{D}$ multi-physical modeler environment. If a $3 \mathrm{D}$ architecture is satisfactory, STs add SAs' requirements that include the different physics requirements, in order to realize the physical 
modeling of the 3D proposed architecture, taking into consideration for example, the thermal radiation, conduction, convection areas and acceptable physical values range of components, etc. Then, STs can do simulations in order to analyze for example the effects of the thermal, electromagnetic and vibrations fields for the given 3D architecture. Based on these results, if they are satisfactory the corresponding 3D architecture is traced back to the SAs in the System model through the reverse model transformation, if they are not satisfactory the Gs propose a new 3D architecture taking into account the physical unsatisfactory constraints, in collaboration with STs. If the Gs cannot succeed in finding a 3D architecture that meets SAs' requirements and multi-physical constraints, the request for a modification is then transmitted to the SAs who are asked to change their proposed physical architecture.

In fact, as the System model is semantically enriched, the physical architecture together with the geometrical and multiphysical requirements is automatically provided, by model transformation, first to Gs so that they can allocate the geometry and position components, and then to STs so that they model the physical behavior. Both of these will realized in a single 3D and Simulation model. This work done by the Gs and the STs is now realized in the same environment using the SAMOS (Spatial Architecture based on Multi-physics and Organization of Systems) platform, eliminating the need for the two model transformations of the second approach, respectively between the System and 3D models, and the System and the Simulation models.

The main advantage of this approach, compared with others, is the unique 3D multi-physical modeler environment that allows to reduce time and cost, by easing the quick evaluation of 3D physical architecture thanks to the direct collaboration between the Gs and the STs, without requiring the SAs' intervention. It also contributes to reducing the time of iterations and thus to finding a 3D physical architecture that meets SAs' requirements and is validated according to its multi-physical behavior.

Finally, the single bidirectional model transformation between the System model and the 3D and Simulation model ensures the consistency and traceability of the design models exchanged in the different steps of the conceptual design, from the specifications until the validation of the successful architecture by the SAs.

As this approach meets, in many aspects, the high industrial expectations previously described, we have chosen this approach to detail how this platform would be implemented.

\section{DESCRIPTION OF THE SAMOS PLATFORM}

\section{A. Global approach}

In order to realize the system architecture, the illustration of the SAMOS (Spatial Architecture based on Multi-physics and Organization of Systems) approach will be done by modeling System models in SysML. Even if the industrial use of this language is often unpopular due to the difficulty for corresponding commercial tools to model complex systems, this language offers some advantages to support this approach. Indeed SysML capacities include the language semantic enrichments through extensions, the possibility of collaborative use and finally the model ability to multi-view while keeping model consistency.

Therefore, first geometrical and multi-physical enrichments will be provided by developing SysML extensions. Indeed, even if model transformation of System model can be processed by using the SysML language without any extension, stereotypes and tags developed in extensions ease the modeling processing. They indeed define the modeling elements available to the users that are necessary to make the model usable for the model transformation. SysML extensions constrain System model designers (SAs) to build a model meeting a structure that agrees with the defined specific metamodel, in order to ensure the success of the model transformation. However, as it is also important that STs and Gs can themselves specify respectively, physical and geometrical, requirements, the reverse model transformation, from the 3D multi-physical architecture model into SysML model, to trace back these new technical requirements is then made possible through the semantic enrichment (specific extensions) of the System model.

The description of the SAMOS platform is presented in Fig. 4. This platform supposes to develop two specific extensions: a multi-physical one relying on a geometrical one, since all physical behaviors are based on specific geometries (of the component and of the physical phenomena).

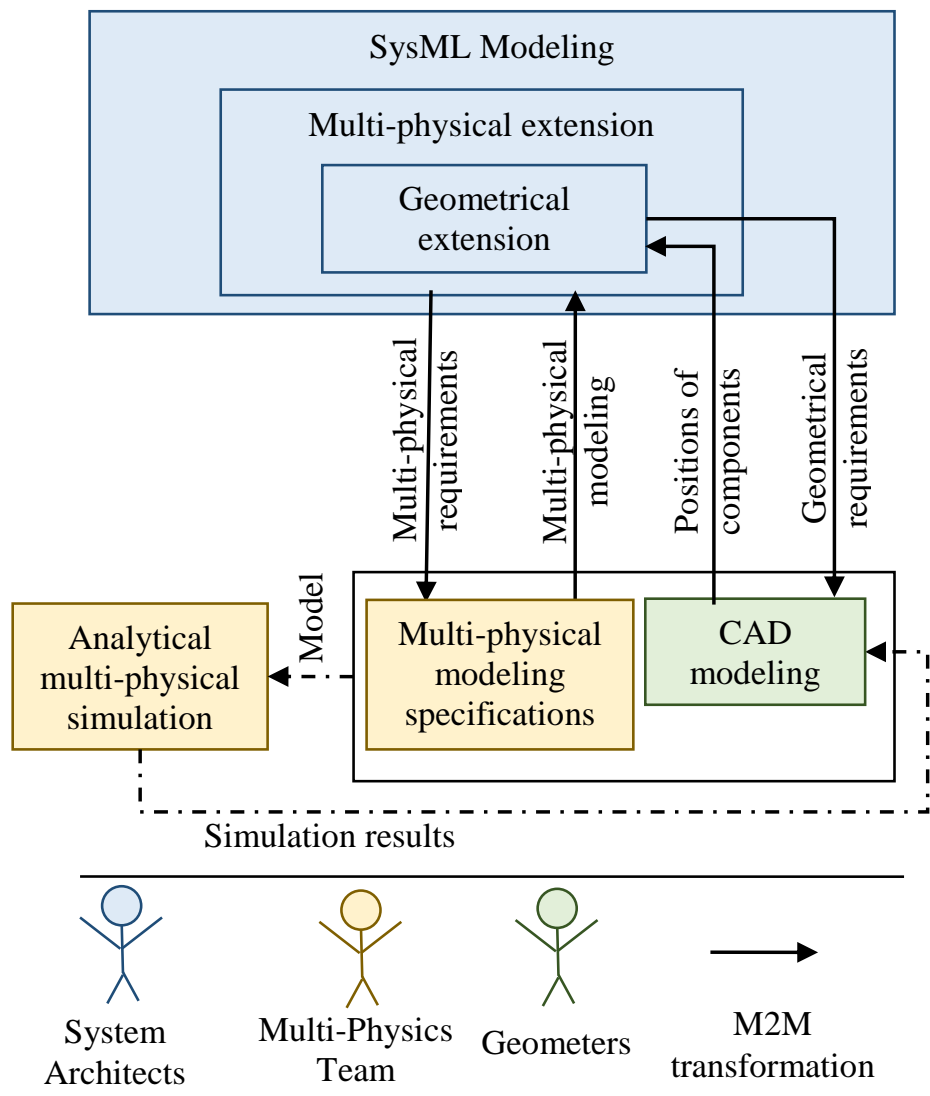

Fig. 4 SAMOS platform implementation. 
To ensure the industrial usability, the System and 3D multiphysical models could be hosted on a shared database, so that all design actors can access it for their collaborative tasks.

\section{B. Geometrical SysML extension}

Geometry is an important part of the development of the SAMOS Platform. As we are in conceptual design, the geometry definition of component requires to be simplified. After having studied numerous available methods to manage geometrical data [16], the most appropriate geometry modeling that we chose is the Technologically and Topologically Related Surfaces (TTRS) approach [17]. Indeed, the management of surfaces is really important, be it for the 3D representation of the system components, or for the multi-physics behaviors that are usually modeled by surface-flows. In this approach, each TTRS belongs to a class according to its kinematic invariance degree (TABLE I). It has a Minimal Reference Geometric Element (MRGE) according to its class. This MRGE enables to place and orientate the TTRS. To position TTRS relatively to each other, a constraint can be added between two TTRSs among the 13 predefined constraints. This geometrical SysML extension has already been developed and detailed in our previous work [16].

TABLE I. DESCRIPTION OF TTRS CLASSES AND ASSOCIATED MRGE

\begin{tabular}{|c|c|c|c|c|c|c|c|}
\hline TTRS & Complex & Prismatic & Revolute & helicoid & cylinder & plan & spherical \\
\hline $\begin{array}{c}\text { Invariance } \\
\text { degree }\end{array}$ & 0 (identity) & 1 translation & 1 rotation & $\begin{array}{c}1 \text { rotation \& } \\
1 \text { translation } \\
\text { combined }\end{array}$ & $\begin{array}{l}1 \text { rotation \& } \\
1 \text { translation }\end{array}$ & $\begin{array}{c}1 \text { rotation \& } \\
2 \text { translations }\end{array}$ & 3 rotations \\
\hline \multirow{3}{*}{ MGRE } & Point & & Point & Point & & & Point \\
\hline & Line & Line & Line & Line & Line & & \\
\hline & Plan & Plan & & & & Plan & \\
\hline
\end{tabular}

\section{Multi-physics SysML extension}

To meet the two MBSE objectives of respectively, design specifications and traceability, and then allow to assess concept architectures under multi-physical constraints, it is necessary to develop a multi-physics SysML extension.

This semantic enrichment of the System model enables for SAs, on one hand, to specify some known multi-physical requirements (notably when redesigning a system), before the model transformation into the 3D simulation model, and on the other hand, to trace the multi-physical modeling specifications defined by the multi-physical team and the simulations results given by the STs. This work done in the conceptual design phase is most valuable and has a high importance for the continuity to the next design phases, such as for the Finite Element Modeling in the detailed design. Indeed, the analytical simulations done at this level and the preliminary generated results taking into account multi-physical requirements, allow to validate the choice of components and their corresponding volume allocation earlier in the design cycle.

Thus, the challenge is now to develop this multi-physical extension including the thermal fields, the electromagnetic fields and the vibrations. For e.g., for the electromagnetic fields, the ElectroMagnetic Compatibility (EMC) [18], the conduction and the radiation modeling will be developed.
Moreover, when developing this new extension, the difficulty will also be to take into consideration the different interactions between the different physics. Each physics shouldn't be studied separately from the others. Thus, the extension has to include the type of the considered coupling, be it weak or strong, and the different influence parameters. For e.g., the thermal and the electromagnetic fields are coupled, and the coupling type differs from an application to another [19] [20] [21]. In SysML, these different coupling equations will be then included in this new extension.

\section{Model transformation rules}

Once the implementation of SysML extensions are finished, transformation rules will be implemented to ensure a seamless link between the System and 3D multi-physical models. Because the model transformations have to be realized from too different semantics, it will be an exogenous (i.e. nonexhaustive) bidirectional model transformation [2]. These model transformations will be described in a further paper.

\section{CONCLUSION}

This paper presents three different MBSE approaches (design interactions management in a new architecture platform; geometrical and multi-physical enrichments for semantic interoperability and SAMOS approach), in order to address design interactions management between the different design actors (System Architects (SAs), Geometers (Gs) and Simulation Teams (STs)), for the concept architecture assessment under geometrical multi-physical constraints, during the conceptual design phase. The last approach (SAMOS) is then detailed, since it decreases design time and costs by reducing the number and time of iterations between previous actors. Then, the different platform elements to be implemented have been presented: the geometrical and multiphysics SysML extensions including the multi-physical couplings considerations, and the model transformation rules. Finally, the SAMOS platform will ensure to find a system concept architecture meeting the different spatial and multiphysical requirements, while reducing modeling time and costs.

\section{ACKNOWLEDGMENT}

A part of this research work has been supported in part by the Technological Research Institute SystemX, and therefore granted with public funds within the scope of the French Program "Investissements d'Avenir".

\section{REFERENCES}

[1] VDI-Richtlinie 2221, "Methodik zum Entwickeln und Konstruieren technischer Systeme und Produkte", VDIVerlag, Düsseldorf, 1993.

[2] Czarnecki, Krzysztof, and Simon Helsen. "Feature-based survey of model transformation approaches." IBM Systems Journal 45.3 (2006): 621-645.

[3] J. Bézivin, F. Büttner M. Gogolla F. Jouault I. Kurtev A. Lindow . Model transformations? transformation models!. In Model driven engineering languages and systems 2006 pp. 440-453.

[4] F. Jouault F. Allilaire, J. Bézivin, and I. Kurtev. "ATL: A model transformation tool." Science of computer programming 72 , no. 1 (2008): 31-39. 
[5] S. Turki, T. Soriano "A SysML extension for Bond Graphs support", IEEE ICTA'05 Thessaloniki, Greece, 15-16 October, 2005.

[6] Y. Cao, Y. Liu, H. Fan, B. Fan, "SysML-based uniform behavior modeling and automated mapping of design and simulation model for complex mechatronics", Computer-Aided Design, Volume 45, Issue 3, March 2013, Pages 764-776.

[7] Sean Hay Kim, "Automating building energy system modeling and analysis: An approach based on SysML and model transformations", Automation in Construction, Volume 41, May 2014, Pages 119-138.

[8] G.-D. Kapos, V. Dalakas, A. Tsadimas, M. Nikolaidou, D. Anagnostopoulos, "Model-based system engineering using SysML: Deriving executable simulation models with QVT," Proceedings Systems Conference (SysCon), 2014 8th Annual IEEE. IEEE, 2014

[9] C. J.J. Paredis, Y. Bernard, R. M. Burkhart, H.-P. de Koning, S. Freidenthal, P. Fritzson, N. F Rouquette, W. Schamai, "An overview of the SysML-Modelica transformation specification", INCOSE 2010.

[10] W. Schamai, P. Fritzson , C. Paredis, A. Pop, "Towards unified system modeling and simulation with ModelicaML: modeling of executable behavior using graphical notations.", Proceedings 7th Modelica Conference, Como, Italy, pp. 612-621, 2009.

[11] M. M. Baysal, U. Roy, R. Sudarsan, R. D. Sriram, K. W. Lyons, « Product Information Exchange Using Open Assembly Model: Issues Related to Representation of Geometric Information », ASME 2005 International Mechanical Engineering Congress and Exposition, pp. 601612. American Society of Mechanical Engineers, 2005.

[12] A. Albers, and C. Zingel "Interdisciplinary Systems Modeling Using the Contact \& Channel-model for SysML." DS 68-9: Proceedings of the 18th International Conference on Engineering Design (ICED 11), Impacting Society through Engineering Design, Vol. 9: Design Methods and Tools pt. 1, Lyngby/Copenhagen, Denmark, (2011).

[13] A. Albers, A. Braun, E. Sadowski, D. F. Wyatt, D. C. Wynn, and P. J. Clarkson. "Contact and Channel Modelling Using Part and Function
Libraries in a Function-Based Design Approach." ASME 2010 International Design Engineering Technical Conferences and Computers and Information in Engineering Conference, pp. 393-404. American Society of Mechanical Engineers, 2010.

[14] A. Albers, and C. Zingel. "Extending SysML for Engineering Designers by Integration of the Contact \& Channel-Approach (C\&C2-A) for Function-Based Modeling of Technical Systems." In CSER, pp. 353362. 2013.

[15] D. Bohnke, A. Reichwein, and S. Rudolph. "Design language for airplane geometries using the unified modeling language." In ASME 2009 International Design Engineering Technical Conferences and Computers and Information in Engineering Conference, pp. 661-670. American Society of Mechanical Engineers, 2009.

[16] R. Barbedienne, O. Penas, J.-Y. Choley, A. Rivière, A. Warniez, F. Della Monica "Introduction of Geometrical Contraints Modeling in SysML for Mechatronic Design", Mecatronics (MECATRONICS), 2014 10th France-Japan/8th Europe-Asia Congress on. IEEE, 2014.

[17] A. Clément, A. Rivière, P. Serré \& C. Valade, "The TTRSs : 13 Constraints for Dimensioning and Tolerancing", Geometric Design Tolerancing: Theories, Standards and Applications ,1998, pp 122 - 121.

[18] Paul, C. R. (2006). Introduction to electromagnetic compatibility (Vol. 184). John Wiley \& Sons.

[19] P. Tixador, G. David, T. Chevalier, Meunier, G., \& Berger, K. (2007). Thermal-electromagnetic modeling of superconductors. Cryogenics, 47(11), 539-545.

[20] D. Carstea, I. Carstea, \& A. Carstea "Numerical simulation of coupled electromagnetic and thermal fields in cable terminations". Telecommunications in Modern Satellite, Cable and Broadcasting Services, 2005. 7th International Conference on. Vol. 2. IEEE, 2005.

[21] J.-P.Bastos, M. F. R. R. Cabreira, N. Sadowski, S. R. Arruda \& S.L. Nau, S. L. (1997). A thermal analysis of induction motors using a weak coupled modeling.Magnetics, IEEE Transactions on, 33(2), 1714-1717. 